\title{
A Snapshot On Socio-economic Status and Outcomes in Oropharyngeal Cancer
}

Authors: Yasmin Abbas MRCS, Zi-Wei Liu FRCS(ORL-HNS), Paul Stimpson FRCS(ORL-HNS), Safina Ali FRCS(ORL-HNS) Affiliations: ENT department, Barts Health NHS Trust, Whitechapel Rd,Whitechapel, London EI IBB

Bartshealth is one of the largest NHS trusts in the UK, with a catchment of 2.5 million people ${ }^{1}$ in East London. It is one of the most ethnically diverse areas as well as most deprived; Tower Hamlets, Hackney and Newham ranking as the top 3 nationally ${ }^{2}$. It is recognized that age, male gender and social deprivation are associated with an increased cancer risk ${ }^{3}$. There is a clear increase in head and neck cancer with oropharyngeal cancers having the most significant rise especially amongst middle-aged adults from highest level of deprivation ${ }^{4}$.

We wanted to determine whether healthcare-seeking behavior is different amongst different ethnic groups, and also whether cancer outcomes can be correlated to indices of multiple deprivation.

\section{Methods:}

This is a retrospective study of oropharyngeal head and neck cancer cases diagnosed between 2014-2016 at a tertiary cancer centre. Patient information was collected including age, diagnosis, staging and treatment outcomes. This was correlated with ethnicity and indices of multiple deprivation. We extracted information about diagnosis and treatment from electronic notes and MDT outcomes in our institution. The English Indices of deprivation 2015 database was used for deprivation data ${ }^{5}$.



\section{Results:}

There were 62 patients diagnosed with oropharyngeal cancer between 2014-2016, of which the ratio of men to women was $3: 1$. The predominant subtypes being the tonsil in 27 patients $(43.5 \%)$ and 25 patients with base of tongue $(40.3 \%)$. Nine $(14.5 \%)$ patients presented with symptoms of their cancer to the emergency services rather than through the 2 week wait pathway via their GP. A Chi-squared test was used to analyse the relationship between ethnicity, deprivation and diagnosis.

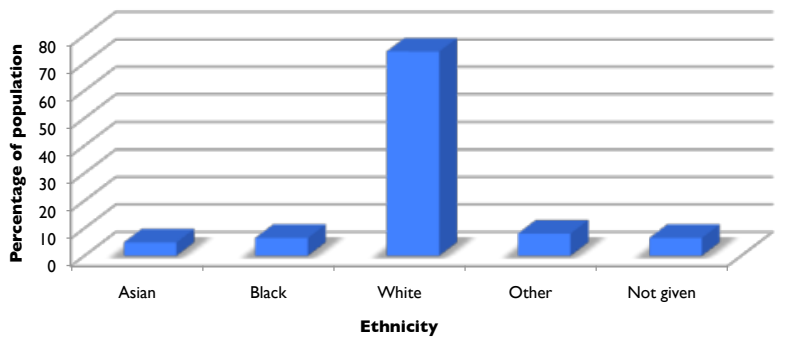

Table I: Ethnicity distribution of oropharyngeal cancers

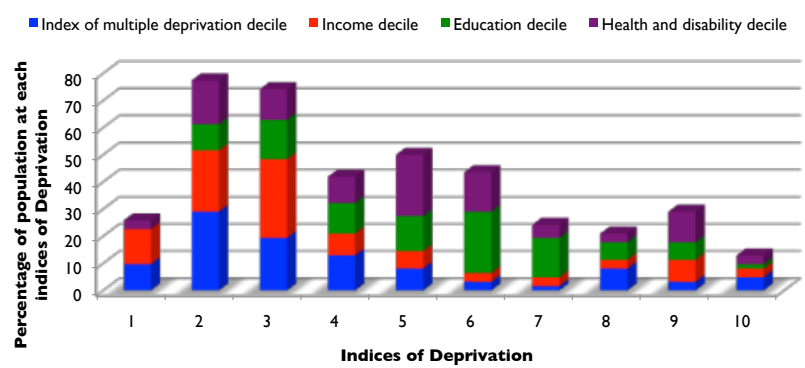

Table 2: Distribution of deprivation within population

\section{Conclusion:}

There is a $3: 1$ male to female ratio, the predominant subtypes were tonsil (43\%)and base of tongue( $40 \%)$. There was no statistical correlation between the different factors, a trend was observed in that women present with an earlier tumour stage. The following factors were associated with a higher incidence of oropharyngeal cancer:
- Male gender
-Tonsil subsite
-56-70 age group
-Moderate education backgrounds
-More deprived backgrounds.

\section{References}

